\title{
Die Demokratie im Zeichen der Coronakrise. Chance oder Bedrohung?
}

Zusammenfassung: Die abnehmende Souveränität der nationalstaatlichen Politik infolge der Globalisierung, wachsende soziale und wirtschaftliche Ungleichheit, die Gefährdung liberaler und demokratischer Prinzipien durch die Digitalisierung sowie das notorische Problem der mangelnden Zukunftsverantwortung setzen die demokratischen Systeme unter Stress. Wie wirkt sich Corona-Pandemie auf diese Problemtendenzen aus? Während die Politik ihren Primat in der Krise zurückgewinnt, droht sie die Ungleichheiten in der Gesellschaft weiter zu verschärfen. Bei der Digitalisierung könnten kurzfristige Rückschläge etwa beim Datenschutz von längerfristigen Vorteilen durch die krisengetriebene Modernisierung der Wirtschafts-, Verwaltungs- und Erziehungstätigkeit aufgewogen werden. Dasselbe gilt für den Klimaschutz, wenn dieser durch eine bessere Risikovorsorge gegen vergleichbare Pandemien mit befördert wird. Die These, dass autoritäre Systeme für die Krisenbewältigung besser gerüstet sind als die Demokratien, lässt sich insgesamt nicht bestätigen.

Schlüsselwörter: Coronakrise, Demokratie, autoritäre Systeme, Globalisierung, Digitalisierung

Frank Decker: Democracy and the Coronavirus Pandemic: Chance or Danger?

Summary: The declining capacities of national governments, increasing social and economic inequality, the undermining of liberal and democratic principles by digitalization and the notorious neglect of future responsibilities are the main problems that place contemporary democracies under stress. How does the coronavirus pandemic affect these tendencies? While the state has regained part of its sovereignty, the crisis is going to dramatically aggravate existing inequalities. Regarding digitalization, short term setbacks (e.g. data protection) might be outweighed by long term benefits through modernization processes in the economic, administrative and educational fields. The same could prove true for climate protection, as the causes of climate change and pandemics such as the coronavirus outbreak partially overlap. All things considered, the assertion that authoritarian systems are superior to democracies in managing crises cannot be confirmed.

Keywords: Coronavirus pandemic, democracy, authoritarian systems, globalization, digitalization 
Frank Decker, Dr. phil., Dipl.-Pol., Professor am Institut für Politische Wissenschaft und Soziologie der Universität Bonn und wissenschaftlicher Leiter der Bonner Akademie für Forschung und Lehre praktischer Politik (BAPP).

Kontakt: frank.decker@uni-bonn.de

Auch wenn der Höhepunkt der Coronakrise erst bevorsteht und ihre Dauer ungewiss bleibt, ist das Nachdenken über die Krisenbewältigung und die langfristigen Folgen der Pandemie in vollem Gange. Die Fragen betreffen dabei auch das politische System. Der Verdacht steht im Raum, dass autoritäre oder weniger freiheitlich verfasste Länder wie China oder Südkorea eher in der Lage seien, solcher Katastrophen Herr zu werden, als die Demokratien des Westens. ${ }^{1}$ Diese können ihre demokratischen und rechtsstaatlichen Prinzipien auch im Ausnahmezustand nicht einfach suspendieren, selbst wenn sie im Kampf gegen die Pandemie hart an deren Grenzen gehen müssen.

\section{Niedergang oder Wandel der Demokratie?}

Die These von der Überlegenheit und besseren Zukunft der Autokratien ist nicht neu. Wenn sie in den letzten Jahren an Popularität gewonnen hat, spricht das nicht unbedingt für ihre empirische Evidenz. Stattdessen reflektiert es die zunehmenden Krisentendenzen im westlichen Gegenmodell, die sich vor allem an der wachsenden Resonanz systemfeindlicher oder -skeptischer Parteien ablesen lassen. Mit der nachholenden Etablierung des Rechtspopulismus ${ }^{2}$ ist nun auch in Deutschland zur Gewissheit geworden, dass die Ära der demokratischen Stabilität, die den Ländern der westlichen Welt jahrzehntelang Wohlstand und Frieden beschert hat, dem Ende zugeht. Andere Länder sind in der demokratischen Regression schon sehr viel weiter. Mit Ungarn und Polen gleiten zwei Mitgliedsstaaten der EU offen in autoritäre Verhältnisse $\mathrm{ab}^{3}$, und in den USA markiert die Präsidentschaft Donald Trumps den vorläufigen Kulminationspunkt einer jahrzehntelangen Erosion demokratischer Strukturen. ${ }^{4}$

1 Vgl. Kishore Mahbubani, Has the West Lost It? A Provocation, London 2018.

2 Vgl. Frank Decker, »Die Ankunft des neuen Rechtspopulismus im Parteiensystem der Bundesrepublik « in: Christoph Bieber u.a. (Hg.), Regieren in der Einwanderungsgesellschaft, Wiesbaden 2017, S. 55-61.

3 Vgl. Manfred Sapper / Volker Weichsel (Hg.), Unterm Messer. Der illiberale Staat in Ungarn und Polen, Berlin 2018 (= osteuropa H. 3-5/2018).

4 Vgl. Patrick Horst / Philipp Adorf / Frank Decker (Hg.), Die USA - eine scheiternde Demokratie?, Bonn 2019. 
Alarmistische Prognosen, die die demokratischen Systeme im »Zerfall «5, »Absterben $\ll^{6}$ oder »auf dem Weg in die Unfreiheit $«^{7}$ sehen, haben daher zur Zeit Konjunktur. Dass die durchaus abwägende Argumentation der Autoren den reißerischen Buchtiteln selten entspricht, ist dabei gleichermaßen symptomatisch und bemerkenswert. Abgesänge auf die Demokratie mit vergleichbaren Krisendiagnosen hat es - zumal mit Blick auf Parteien, Regierungen und Parlamente - schon immer gegeben. Auch heute haben wir es eher mit einem - allerdings grundlegenden - Wandel zu tun als mit einem Niedergang. In diesem Wandel spiegeln sich neue und neuartige Herausforderungen des Regierens, die Akteure und Institutionen unter wachsenden Druck und damit das demokratische System insgesamt unter Stress setzen. Die Herausforderungen und Probleme lassen sich in vier Stichworten beschreiben. ${ }^{8}$

Souveränität. Das erste und schwierigste Problem besteht darin, dass die beschleunigte Globalisierung der Realwirtschaft und der Finanzmärkte den Handlungsspielraum der nationalstaatlichen Politik zunehmend einschränkt. ${ }^{9}$ Dies betrifft vor allem die unter Legitimationsgesichtspunkten besonders wichtigen Bereiche der Daseinsvorsorge und Sozialpolitik. Die Staaten können zwar durch internationale Kooperation Gestaltungsmacht zurückgewinnen, doch ändert das nichts daran, dass die Demokratie als Regierungs- und Herrschaftsform an die nationale Sphäre gebunden bleibt. Wie mühselig es ist, sie über diese Sphäre hinauszuheben, zeigen der Verlauf und die aktuellen Schwierigkeiten des europäischen Integrationsprozesses. ${ }^{10}$

Ungleichbeit. Eng verwoben damit ist das zweite Problem. Indem sie die nationalen Wohlfahrtsstaaten und Arbeitsmärkte unter Druck setzt, verstärkt die Globalisierung die wirtschaftliche und soziale Ungleichheit. ${ }^{11}$ Während das obere Drittel der Gesellschaft Wohlfahrtsgewinne einstreicht, steigt das unter Drittel ab oder fühlt sich von Abstieg bedroht. Verschärft wird die Ungleichheit durch den gleichzeitig rückläufigen gemeinschaftlichen Zusammenhalt, der eine Folge der Individualisierung darstellt und auch die Alltagskultur dem Prinzip des Marktes immer mehr unterwirft. ${ }^{12}$ Als kon-

5 Sascha Mounk, Der Zerfall der Demokratie. Wie der Populismus den Rechtsstaat bedroht, München 2018.

6 Steven Levitsky / Daniel Ziblatt, Wie Demokratien sterben. Und was wir dagegen tun können, Frankfurt a.M. 2018.

7 Timothy Snyder, Auf dem Weg in die Unfreibeit. Russland, Europa, Amerika, München 2018.

8 Vgl. Frank Decker, "Herausforderungen der repräsentativen Demokratie« in: Andreas H. Apelt / Dirk Reimers (Hg.), Repräsentative versus direkte Demokratie, Halle (Saale) 2018, S. $123 \mathrm{ff}$.

9 Vgl. Lea Heyne, »Globalisierung und Demokratie. Führt Denationalisierung zu einem Verlust an Demokratiequalität?« in: Wolfgang Merkel (Hg.), Demokratie und Krise, Wiesbaden 2015, S. 277-305.

10 Vgl. Jürgen Rüttgers / Frank Decker (Hg.), Europas Ende, Europas Anfang. Neue Perspektiven für die Europäische Union, Bonn 2017.

11 Vgl. Anthony B. Atkinson, Ungleichheit. Was wir dagegen tun können, Stuttgart 2016; Branko Milanović, Die ungleiche Welt. Migration, das Eine Prozent und die Zukunft der Mittelschicht, Berlin 2016; Thomas Piketty, Das Kapital im 21. Jabrbundert, München 2014.

12 Vgl. Andreas Reckwitz, Die Gesellschaft der Singularitäten. Zum Strukturwandel der Moderne, Berlin 2017. 
fliktträchtig erweist sich besonders die Inklusion der nicht eingesessenen Bevölkerungsminderheiten und Zuwanderer. (In anderen Bereichen - etwa bei der Geschlechtergleichheit oder den Rechten sexueller Minderheiten - sind dagegen bedeutende Gleichheitsfortschritte zu verzeichnen, weshalb man sich hüten sollte, die 1950er oder 1960er Jahre als "goldenes Zeitalter« der Demokratien nostalgisch zu überhöhen.)

Digitalisierung. Das dritte große Problem entsteht durch die Digitalisierung. Es ist in seinen Ausprägungen und Konsequenzen noch nicht wirklich absehbar. Der von manchen geäußerten Hoffnung, das Netz führe zu mehr direkter Demokratie und einer größeren Entscheidungstransparenz, stellen andere die Schattenseiten einer immer stärker fragmentierten Öffentlichkeit und der Unterminierung elementarer Prinzipien wie Respekt und Dialogbereitschaft entgegen, auf denen das Funktionieren der Demokratie beruhe. ${ }^{13}$ Noch gravierendere Folgen drohen durch die Aushöhlung individueller Freiheitsrechte (Datenschutz) oder manipulative Eingriffe in die Freiheit des demokratischen Meinungs- und Willensbildungsprozesses mittels sogenannter Social Bots und anonymisierter Netzwerke, die als Mittel der stillen Kriegführung auch von Terroristen oder auswärtigen Mächten eingesetzt werden können.

Zukunftsverantwortung. Das vierte Problem der Demokratie ist ein notorisches - es liegt in der Bevorzugung von gegenwärtigen gegenüber künftigen Interessen. Wo das Stimmrecht bei den Lebenden liegt, geraten die Belange der späteren, noch nicht geborenen Generationen systematisch aus dem Blick. ${ }^{14}$ Die Brisanz dieses Problems lässt sich heute vor allem an zwei Bereichen festmachen: der privaten und öffentlichen Verschuldung, die die Krisenanfälligkeit der Wirtschaft erhöht und gleichzeitig - durch die Abhängigkeit der nationalen Politik von den internationalen Finanzmärkten - das Souveränitätsproblem verstärkt, und den ökologischen Gefährdungen im Zuge der Erderwärmung und des Artensterbens.

Welche mittel- und langfristigen Folgen die Coronakrise für die Demokratie hat, darüber scheiden sich unter den politischen und wissenschaftlichen Beobachtern schon jetzt die Geister. Während die einen eine Verschärfung der Problemtendenzen befürchten, die die ohnehin brüchige Legitimation der demokratischen Systeme weiter untergrabe, sehen die anderen in der Pandemie eine Chance, Fehlentwicklungen zu korrigieren und die Demokratie - national, europäisch und international - auf eine neue, gesichertere Basis zu stellen. Um zu prüfen, welches Szenario das wahrscheinlichere ist, gilt es die beschriebenen vier Herausforderungen durch die Brille der Coronakrise im einzelnen zu betrachten.

13 Vgl. z. B. Henrik Müller, Kurzschlusspolitik. Wie die permanente Empörung unsere Demokratie zerstört, München 2020; Timothy Garton Ash, Redefreiheit. Prinzipien für eine vernetzte Welt, München 2016.

14 Vgl. Tine Stein, »Zum Problem der Zukunftsfähigkeit der Demokratie« in: Bernward Gesang (Hg.), Kann Demokratie Nachhaltigkeit?, Wiesbaden 2014, S. 47-63; Peter Graf Kielmansegg, »Können Demokratien zukunftsverantwortlich handeln? « in: Merkur 57 (2003) H. 7, S. 584-594. 


\section{Ein neuer Primat der Politik}

Was das Souveränitätsproblem angeht, dreht die Krise die mit der Globalisierung einhergehenden Tendenzen der Entdemokratisierung insofern zurück, als sie den Primat der Politik wiederherstellt beziehungsweise die Wirtschaft diesem Primat nahezu vollständig unterwirft. Der Hinweis, dass an dessen Stelle nun der Primat der Wissenschaft, sprich der Virologie getreten sei, geht fehl, weil die ohnehin uneinheitlichen Empfehlungen der Wissenschaftler die politischen Verantwortungsträger von der Notwendigkeit, verschiedene Ziele gegeneinander abzuwägen, nicht entbinden. Diese Abwägungen lassen stets Raum für Alternativen. Deshalb gewinnen das Parlament und eine funktionierende Medienöffentlichkeit in der Krise sogar an Bedeutung - trotz der sprichwörtlichen »Stunde der Exekutive«, die in Ausnahme- und Notstandssituationen schlägt. ${ }^{15}$ Auch über die Grenzen, die der Rechts- und Verfassungsstaat dem Handeln der Regierenden zieht, muss politisch gestritten und wenn nötig von Gerichten entschieden werden.

Die Gefahr, dass die Regierungen die Notsituationen ausnutzen, um ihre Befugnisse über die Dauer der Krise hinaus auszudehnen, ist potenziell natürlich stets gegeben. Sie dürfte aber dort, wo autoritäre Dispositionen bereits bestehen, ungleich größer sein als in den konsolidierten rechtsstaatlichen Demokratien. Insbesondere Ungarn unter Viktor Orbán gibt hier ein warnendes Beispiel. ${ }^{16}$ Während die Wahrscheinlichkeit der Rückkehr zu normalen Verhältnissen in den etablierten Demokratien hoch ist, könnten auf der anderen Seite gerade die autoritären oder totalitären Systeme durch die Krise destabilisiert werden. Die fehlende Meinungs- und Pressefreiheit, die zur raschen Ausbreitung der Seuche an deren Ursprungsort Wuhan beitrug, hat das Misstrauen der chinesischen Bevölkerung in ihre politische Führung erhöht. ${ }^{17} \mathrm{Ob}$ die Menschen dem Regime den von der Propaganda verkündeten Sieg über das Virus tatsächlich abnehmen, lässt sich bezweifeln.

Was die Globalisierung betrifft, ist ihre Rolle in der Krise ambivalent. Einerseits ist sie mitursächlich für die Entstehung und rasche globale Ausbreitung des Virus und ein Hemmnis für die nationale Politik bei der autonomen Gefahrenabwehr und Krisenbewältigung. Andererseits versetzt sie uns - durch den wissenschaftlichen und technologischen Austausch - in die Lage, mit solchen Epidemien heute viel besser fertig zu werden als in früheren Epochen der Weltgeschichte, man denke etwa an die mittelalterliche Pest oder die nach dem Ersten Weltkrieg wütende Spanische Grippe. ${ }^{18}$ Niemand zweifelt zum Beispiel daran, dass es zur Entwicklung eines Impfstoffs gegen das Virus kommen wird - die Frage lautet allenfalls, wann dieser zur Verfügung steht und wie

15 Vgl. Christoph Schönberger / Sophie Schönberger, »Regiert bald ein Notausschuss?« in: Frankfurter Allgemeine Zeitung vom 26. März 2020.

16 Vgl. Silviu Mihai, »Auf dem Weg in eine Diktatur« in: Die Zeit vom 31. März 2020.

17 Vgl. Lea Deuber, »Wenn Misstrauen viral wird « in: Süddeutsche Zeitung vom 23. Januar 2020.

18 Vgl. Christoph David Piorkowski, »Die Mutter der modernen Pandemien« in: Der Tagesspiegel vom 20.03.2020. 
schnell er weltweit zur Anwendung kommt. Dasselbe gilt für die Bewältigung der wirtschaftlichen Folgen. Ob sie ähnlich rasch gelingt wie nach der Finanzkrise 2008, wird vor allem davon abhängen, wie stark die von der Pandemie bisher noch weniger betroffenen Entwicklungs- und Schwellenländer in den globalen Abwärtssog mit hinein geraten. Die bereits im europäischen Kontext schwierige Solidaritätsfrage bekommt hier noch einmal eine ganz andere Dimension.

Der Rückzug auf das Nationale war und ist in einer Situation der unmittelbaren Bedrohung ein verständlicher Reflex. Das gilt auch für die Mitgliedsstaaten der Europäischen Union, wo dies naturgemäß Unbehagen ausgelöst und tatsächlich zu vielen unschönen Begleiterscheinungen - etwa den unabgestimmt vorgenommenen Grenzschließungen - geführt hat. Dass die EU deshalb geschwächt aus der Krise hervorgehen wird, ist nicht ausgemacht. Dafür muss sie freilich die richtigen Lehren ziehen. Zum einen gilt es sich für vergleichbare Situationen in Zukunft besser zu wappnen: durch eine Zurückholung »systemrelevanter « Produktionen in der Pharmazie und Medizintechnik, Diversifizierung von Lieferketten, Bevorratung von Schutzmaterial und Medikamenten. Dafür könnte Brüssel den Mitgliedsstaaten entsprechende Vorgaben machen. Zum anderen braucht es die Unterstützung der wettbewerbsschwächeren südeuropäischen Länder bei der Bewältigung der sich durch die Krise auftürmenden wirtschaftlichen Probleme und Finanzlasten. Die hartherzige Haltung, mit der Deutschland und die Niederlande sich der Forderung nach einer zeitlich und sachlich begrenzten Vergemeinschaftung von Schulden (»Coronabonds«) entgegengestellt haben, wird mittlerweile auch von vielen Ökonomen hierzulande kritisiert und kann nicht das letzte Wort bleiben. ${ }^{19}$

\section{Wie stark wächst die Ungleichheit?}

Die mangelnde Solidarität auf europäischer Ebene dürfte auch der Sorge entspringen, dass es bereits im nationalen Rahmen schwer genug werden wird, die mit der Coronakrise verbundenen sozialen und wirtschaftlichen Verwerfungen halbwegs erträglich abzufedern. Die Pandemie könnte bestehende Ungleichheiten in der Gesellschaft weiter verschärfen und neue entstehen lassen. Was das Schutzgut Leben und Gesundheit angeht, ist das Virus gerade nicht der Gleichmacher, den Ulrich Beck mit seinem bekannten Satz »Not ist hierarchisch, Smog ist demokratisch « als charakteristisches Merkmal der Risikogesellschaft (im Vergleich zur klassischen Industriegesellschaft) beschrieben hat. ${ }^{20}$ Hauptbetroffen sind die ohnehin gefährdeten Gruppen - Ältere, Pflegebedürftige, Menschen mit Behinderungen und Vorerkrankungen. Durch ihre dem eigenen Schutz dienende Isolation haben sie jetzt zugleich in sozialer Hinsicht die größte Last der Pandemie zu tragen. Die seelischen Folgen, die das für die Menschen selbst und ihre Angehörigen hat, sind kaum zu ermessen.

19 Vgl. Henrik Enderlein, »Jeder stirbt für sich allein« in: Der Spiegel vom 11. April 2020.

20 Ulrich Beck, Risikogesellschaft. Auf dem Weg in eine andere Moderne, Frankfurt a.M. 1986, S. 48 . 
In der Gesellschaft insgesamt fehlt es dabei keineswegs an Mitgefühl, im Gegenteil. Nicht nur, dass die Bürger die ihnen auferlegten strengen Kontaktbeschränkungen bereitwillig akzeptiert haben und diszipliniert einhalten. Viele engagieren sich darüber hinaus, indem sie für ältere Menschen einkaufen oder sich den systemrelevanten Branchen als Arbeitskräfte zur Verfügung stellen. Beides zeugt von einem in dieser Form bisher nicht erlebten Zusammengehörigkeitsgefühl. Vor der Gefahr des Virus fühlen sich die Menschen wie in einer Schicksalsgemeinschaft miteinander verbunden. Wie belastbar diese Verbundenheit ist, wenn es um die Bewältigung der sozialen und ökonomischen Kosten der Pandemie geht, muss sich aber erst noch erweisen.

Tatsächlich sind die Gräben, die die Krise in dieser Hinsicht aufreißt, immens. Die Beschäftigen in der Pflege, im Lebensmittelhandel und in der Logistik, die »an der Front « stehen und sich als Angehörige der systemrelevanten Berufe jetzt unvermittelt einer neuen Wertschätzung erfreuen, arbeiten häufig in prekären Verhältnissen und zu niedrigen Löhnen. Die meisten von ihnen sind Frauen. Auf der Verliererseite befinden sich zugleich viele Alleinerziehende und Familien mit Kindern, die ihren Nachwuchs zu Hause betreuen müssen, solange Schulen und Kitas geschlossen bleiben. Das ohnehin große Bildungsgefälle wird dadurch weiter vergrößert - mit erheblichen Langzeitfolgen. Ähnlich groß sind die Verwerfungen auf dem Arbeitsmarkt. Während die einen über ein sicheres Beschäftigungsverhältnis - etwa im Öffentlichen Dienst - verfügen und von zu Hause aus arbeiten können, müssen die in den ganz oder teilweise stillgelegten Branchen Beschäftigten in Kurzarbeit gehen und vielleicht sogar um ihren Job fürchten. Auch das Privileg des Homeoffice bleibt nur einer Minderheit vergönnt. Der größere Teil der Menschen arbeitet weiterhin im Büro oder Betrieb und setzt sich dort und auf dem Weg dorthin zugleich einem höheren Infektionsrisiko aus.

$\mathrm{Ob}$ und wie lange man sich an die Ungerechtigkeiten erinnern und welche Folgerungen man daraus ziehen wird, wenn die Pandemie hinter uns liegt, bleibt eine offene Frage. Dasselbe gilt für den Abtrag der finanziellen Folgelasten der Krise, die die Staaten jetzt dazu zwingt, sich in einer historisch einmaligen Größenordnung kurzfristig neu zu verschulden. Ausgabenkürzungen und Steuererhöhungen sind damit vorprogrammiert und dürften erhebliche Verteilungskonflikte nach sich ziehen. Diese ließen sich allenfalls durch eine rasche wirtschaftliche Erholung abmildern, die aber umso unwahrscheinlicher ist, je länger der Lockdown andauert. Die politische Polarisierung, die in den letzten Jahren vor allem den Rechtspopulisten in die Hände gespielt hat, könnte dadurch weiter zunehmen.

\section{Chancen und Risiken der Digitalisierung}

Die Digitalisierung erweist sich für die Bewältigung der Krise in vielerlei Hinsicht als Segen. Man stelle sich vor, eine solche Pandemie hätte die Welt in den 1980er oder 1990er Jahren getroffen - ihre medizinische Bekämpfung hätte sich schwieriger gestaltet und die sozialen und ökonomischen Folgen eines Lockdowns wären viel gra- 
vierender gewesen. ${ }^{21}$ Dass ein beträchtlicher Teil der wirtschaftlichen-, Verwaltungsund Erziehungstätigkeit (im Bildungswesen) vom heimischen Computer aus geleistet werden kann, hilft uns jetzt enorm. Es rächen sich aber auch die Versäumnisse: Gerade im Verwaltungs- und Bildungsbereich wäre noch viel mehr Kompensation möglich, hätte man die digitale Modernisierung in den letzten Jahren ehrgeiziger betrieben. Dass diese Modernisierung mit und nach der Krise umso rascher erfolgen und es in Gesellschaft, Staat und Wirtschaft kein Zurück mehr in die analoge Welt vor Corona geben wird, zeichnet sich als eine der wichtigsten Langzeitwirkungen der Pandemie schon heute ab.

Blickt man auf die demokratischen und rechtsstaatlichen Aspekte, so hat die Coronakrise die bekannten Licht- und Schattenseiten der Digitalisierung von neuem offenbart. Einerseits fördert das Netz soziales Engagement und Hilfsbereitschaft, ermöglicht es den Austausch, wo ein physisches Zusammentreffen nicht mehr stattfinden kann. Andererseits bleibt es ein notorischer Tummelplatz für Betrüger und die Verbreiter von Falschnachrichten. Auch das Datenschutzthema erhält durch die Möglichkeit, die Ausbreitungswege des Virus mithilfe von Tracing-Apps nachzuverfolgen, neue Brisanz. Gleichzeitig macht es die bleibende Abhängigkeit der europäischen Länder von den US-amerikanischen Internet-Giganten deutlich. Hier endlich ein Gegenwicht aufzubauen, könnte als industriepolitische Aufgabe der EU eine weitere mittelund langfristige Lehre aus der Krise sein.

Was die unmittelbaren politischen Wirkungen betrifft, hat Corona - wenn der Eindruck nicht täuscht - den Zerfallsprozess der demokratischen Öffentlichkeit zumindest vorübergehend gestoppt. So wie sich die Bürger in der Notsituation wieder verstärkt den Regierenden zuwenden, gewinnen die traditionellen Medien an Vertrauen zurück. Die prinzipiellen Fragen, welche Regeln in der digitalen Welt gelten und wie diese national, in der EU und global durchgesetzt werden können, bleiben aber im Raum und werden die Agenda der Verfassungs- und Demokratiepolitik in den kommenden Jahren weiter prägen.

\section{Auf dem Weg in eine nachhaltigere Zukunft?}

In einer Antwort auf eine parlamentarische Anfrage hatte die Bundesregierung (in Gestalt des Robert-Koch-Instituts) das Szenario einer Coronavirus-Pandemie bereits im Jahre 2012 mit - aus heutiger Sicht - bedrückender Exaktheit beschrieben. ${ }^{22}$ Dass dies politisch folgenlos geblieben ist, mag zum einen mit der angenommenen Todeszahl von 7,5 Millionen innerhalb von drei Jahren (allein in Deutschland und für den Fall, dass keine Gegenmaßnahmen ergriffen werden) zu tun haben, die außerhalb jeglicher

21 Für eine umfassende Bestandsaufnahme vor der Coranakrise vgl. Wolfgang Schroeder / Ursula Bitzegeio / Sandra Fischer (Hg.), Digitale Industrie - Algorithmische Arbeit - Gesellschaftliche Transformation, Bonn 2020 (i.E.).

22 Bundestags-Drucksache 17/12051. 
Vorstellungskraft lag. Zum anderen wurde ein sehr geringe Eintrittswahrscheinlichkeit (»einmal in einem Zeitraum von 100 bis 1.000 Jahren«) unterstellt.

Der in dem Szenario ebenfalls richtig angenommene Ursprungsort des Virus in Südostasien scheint die Theorie zu widerlegen, dass autoritär verfasste Systeme besser in der Lage seien, zukunftsverantwortlich zu handeln, als Demokratien. Die Theorie könnte sogar ganz falsch sein, wenn man bedenkt, dass auch - und vielleicht gerade dort, wo die Regierenden nicht auf die unmittelbare Zustimmung der Regierten angewiesen sind, die Ergebnisse der Politik eine wesentliche Legitimationsgrundlage ihres Handelns und damit des Systems insgesamt bilden. Wenn »gute« Politik darin besteht, für Sicherheit und materiellen Wohlstand zu sorgen, so dürfte besonders das zuletzt genannte Ziel dazu verleiten, Risiken und Gefährdungen hinzunehmen, die mit Blick auf andere Schutzgüter nur schwer hinnehmbar sind. Weil sie solche Zielkonflikte nicht offen benennen und politisch austragen, sind die autoritären den demokratischen Systemen gegenüber hier grundsätzlich im Nachteil. ${ }^{23}$

Dennoch bleibt die Bilanz der Demokratien in Sachen zukunftsgerichteter Politik schlecht. Am greifbarsten ist dies beim Klimaschutz, der ein wahres Menschheitsthema und eine politische Jahrhundertaufgabe darstellt. Unter Wissenschaftlern gibt es die nahezu einhellige Meinung, dass bei einer Temperaturerhöhung von mehr als zwei Grad dramatische Folgen auf die Weltbevölkerung zukommen werden. Um sie abzuwenden, bleibt nur ein relativ schmales Zeitfenster von knapp zwanzig Jahren. Gemessen daran sind die heutigen Anstrengungen sowohl auf der Zielebene (der Reduktionsverpflichtungen) als auch bei der Umsetzung deutlich zu gering. Ob sie durch die Coronakrise einen Schub erhalten werden, wie viele es hoffen und manche erwarten, ist keineswegs sicher.

Die Vehemenz, mit der der Staat zur Zeit in das Wirtschaftsgeschehen eingreift, um die Pandemie einzudämmen, wird von Befürwortern eines strengeren Klimaschutzes als positives Signal gewertet. Wenn man handeln müsse und wolle, so wird insinuiert, könne man das auch. Die scheinbar griffige Parallele geht an den Unterschieden zwischen beiden Krisen jedoch vorbei. Zum einen stellt das Coronavirus eine zwar »unsichtbare «, aber doch manifeste Gefahr dar. Es bedroht nicht nur statistische, sondern ganz konkrete Menschenleben und lässt sich deshalb weder individuell noch politischgesellschaftlich verdrängen. Beim Klimawandel handelt es sich demgegenüber um eine zeitlich versetzt auftretende, »schleichende « Katastrophe, deren Folgen - wie drastisch sie auch ausgemalt werden - uns heute noch eher abstrakt vorkommen. ${ }^{24}$ Zum anderen sind die Ursachen der Katastrophe und damit auch die Bekämpfungsmöglichkeiten in ihrer Komplexität grundverschieden. Reicht es bei Corona aus, einen Impfstoff zu finden und - bevor das der Fall ist - die Ausbreitung der Seuche durch eine Reduktion der Ansteckungsmöglichkeiten zu bremsen, erfordert die Klimaneutralität eine umfas-

23 Für einen empirischen Leistungsvergleich demokratischer und autoritärer Systeme vgl. Manfred G. Schmidt, Demokratietheorien. Eine Einführung, 6. Aufl., Wiesbaden 2019, S. 489 ff.

24 Vgl. bereits Carl Böhret, Folgen. Entwurf für eine aktive Politik gegen schleichende Katastrophen, Opladen 1990, S. 64 ff. 
sende Transformation von Wirtschaft und Gesellschaft. Die durch sie auf den Plan gerufenen Gegenkräfte sind deshalb ungleich massiver. Während die Überwindung der Seuche am Ende nur eine Frage der Zeit (und der Kosten) ist, könnte die globale Gemeinschaft an der Herausforderung des Klimaschutzes sehr wohl scheitern.

Sollte es eine der Lehren der Krise sein, dass die internationale Gemeinschaft und die Staaten im einzeln dem Ausbrauch vergleichbarer Seuchen in Zukunft besser vorbeugen, wäre das zugleich eine positive Nachricht für die Klimapolitik. Beide Katastrophen sind nämlich gleichermaßen menschengemacht und in ihren Ursachen wenn man etwa an die Zurückdrängung des Lebensraums von Tieren und Pflanzen denkt - zum Teil identisch. ${ }^{25}$ Jüngste Untersuchungen zeigen zudem, dass die Sterblichkeit durch das Virus in Regionen mit starker Feinstaubbelastung besonders hoch ist $^{26}$ - auch hier verstärken sich die Umweltrisiken also gegenseitig. Vor diesem Hintergrund wäre es fahrlässig, die notwendige Ankurbelung der Wirtschaft nach dem Lockdown mit Forderungen nach einer Lockerung der Umwelt- und Klimaschutzziele zu verknüpfen. Die Erfahrung der Verwundbarkeit, die die Pandemie in unser Leben und die gesamte Gesellschaft zurückgeholt hat, könnte und sollte stattdessen der Beginn einer Epoche der Nachhaltigkeit sein. Dass es eine Rückkehr in die Sorglosigkeit des »immer schneller, höher und weiter« der Vor-Corona-Zeit nicht geben wird, erscheint ziemlich gewiss.

25 Vgl. Philip Bethge, »Der Mensch ist schuld an Covid-19« in: Der Spiegel vom 3. April 2020.

26 Vgl. Joachim Müller-Jung, »Verschärft schmutzige Luft das Pandemie-Desaster?« in: Frankfurter Allgemeine Zeitung vom 16. April 2020. 